\title{
Scaling of the critical temperature and the quark potential with a renormalization group improved $\mathrm{SU}(3)$ gauge action *
}

\author{
Y. Iwasakia, ${ }^{a, b}$ K. Kanaya, ${ }^{a, b}$ T. Kaneko, and T. Yoshiéa,b \\ ${ }^{a}$ Center for Computational Physics, University of Tsukuba, Ibaraki 305, Japan \\ ${ }^{\mathrm{b}}$ Institute of Physics, University of Tsukuba, Ibaraki 305, Japan
}

\begin{abstract}
We study the scaling property of the ratio of the critical temperature $T_{c}$ to the square root of the string tension $\sigma$ in the $\mathrm{SU}(3)$ pure gauge theory using a renormalization group (RG) improved action. We first determine the critical coupling $\beta_{c}$ on lattices with temporal extension $N_{t}=4$ and 6 , and then calculate the static quark potential at the critical couplings on lattices at zero temperature. The values of $T_{c} / \sqrt{\sigma}$ in the infinite volume limit are identical within errors, while they are slightly larger than the value extrapolated to the continuum limit with the standard action. We also note that the rotational invariance of the static quark potential is remarkably restored in the both cases, and that the potential $V(R)$ in physical units scales in the whole region of $R$ investigated.
\end{abstract}

\section{Introduction}

In studies of lattice QCD, it is important to control and reduce finite lattice spacing effects. Several improved actions have been proposed for this purpose and some of them have been tested for the scaling behavior of the critical temperature $T_{c}$ [1]. In this work we study the scaling property of $T_{c} / \sqrt{\sigma}$ using a RG improved action proposed by one of the authors [2]:

$S_{g}=1 / g^{2}\left\{c_{0} \sum\right.$ (plaquette $)+c_{1} \sum(1 \times 2$ loop $\left.)\right\}$

with $c_{1}=-0.331$ and $c_{0}=1-8 c_{1}$.

The outline of the calculation of $T_{c} / \sqrt{\sigma}$ is as follows. First we determine the critical coupling $\beta_{c}$ 's on $12^{3} \times 4$ and $18^{3} \times 6$ lattices. Then the string tensions at the two $\beta_{c}$ 's are evaluated from smeared Wilson loops on $12^{3} \times 24$ and $18^{3} \times 36$ lattices, respectively. The string tensions in the infinite volume limit are estimated for the both cases, with a finite size scaling analysis applied using the result of $\beta_{c}$ on a $16^{3} \times 4$ lattice.

\section{Determination of $\beta_{c}$}

The critical couplings $\beta_{c}$ 's for the finite temperature phase transition in the $S U(3)$ pure gauge theory are determined as the location of the peak

\footnotetext{
*Talk presented by T. Kaneko at Lattice 96.
}

Table 1

Parameters of the finite temperature simulations

\begin{tabular}{ccc}
\hline$\beta$ & sweep & thermalization \\
\hline $12^{3} \times 4$ & & \\
2.250 & 12000 & 2000 \\
2.275 & 125000 & 40000 \\
2.300 & 10000 & 1500 \\
\hline $16^{3} \times 4$ & & \\
2.283 & 220000 & 40000 \\
2.290 & 240000 & 40000 \\
\hline $18^{3} \times 6$ & & \\
2.5000 & 120000 & 15000 \\
2.5125 & 256000 & 50000 \\
2.5250 & 210000 & 60000 \\
2.5375 & 135000 & 5000 \\
\hline
\end{tabular}

of the susceptibility of the $Z(3)$ rotated Polyakov line. Parameters of the simulations are summarized in Table 11. Gauge fields are updated by Cabibbo-Marinari-Okawa pseudo-heat bath algorithm here and also for the calculation of the string tension. The results of the susceptibility with the histogram method applied on the $12^{3} \times 4$ lattice are shown in Fig. 1. The error of $\beta_{c}$ is determined by the jack knife method with bin size of 3000 sweeps for the $12^{3} \times 4$ and $18^{3} \times 6$ lattices and 6000 sweeps for the $16^{3} \times 4$ lattice. We have checked that the errors are stable for the bin size 


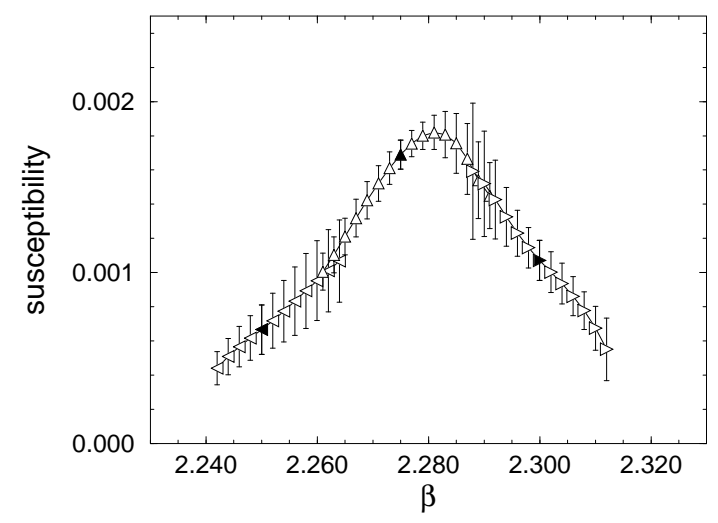

Figure 1. Susceptibility on the $12^{3} \times 4$ lattice. Filled symbols represent the location of simulation points.

larger than these values. We obtain

$\beta_{c}= \begin{cases}2.2827(16) & \left(12^{3} \times 4\right) \\ 2.2863(10) & \left(16^{3} \times 4\right) \\ 2.5157(7) & \left(18^{3} \times 6\right)\end{cases}$

\section{Evaluation of the string tension}

We evaluate the string tension on a $12^{3} \times 24$ $\left(18^{3} \times 36\right)$ lattice at $\beta=\beta_{c}$ for the $12^{3} \times 4$ $\left(18^{3} \times 6\right)$ lattice. After at least 5000 thermalization sweeps, we measure Wilson loops at every 200 sweeps. The spatial paths of the loops are taken to be multiples of spatial vectors $(1,0,0),(1,1,0),(2,1,0),(1,1,1),(2,1,1),(2,2,1)$. The number of configurations is $200(100)$ at $\beta_{c}$ for the $12^{3} \times 4\left(18^{3} \times 6\right)$ lattice. We carry out the smearing technique proposed in Ref. [3] up to 30 (40) times on the $12^{3} \times 24\left(18^{3} \times 36\right)$ lattice. The potential $V(R)$ and the overlap function $C(R)$ are extracted by fitting the Wilson loops to

$W(R, T)=C(R) \exp [-V(R) \cdot T]$.

The optimized smearing time is determined from the condition $C(R) \simeq 1.0$. The string tension is determined by fitting $V(R)$ to

$V(R)=V_{0}-\alpha / R+\sigma R$.
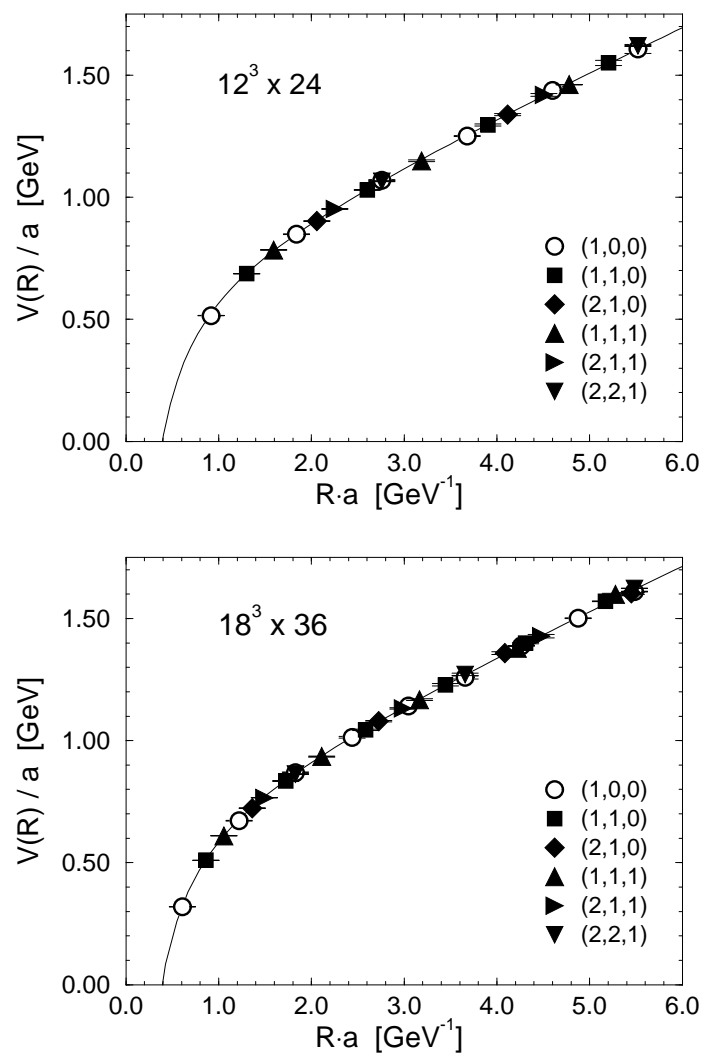

Figure 2. Data for the potential and its fitting curve on the $12^{3} \times 24$ (above) and $18^{3} \times 36$ (below) lattices.

We make correlated fits for the both fits. Fitting ranges are

$$
\begin{array}{lll}
T=3-5 & R=\sqrt{6}-4 \sqrt{2} & \left(12^{3} \times 24\right) \\
T=4-7 & R=2 \sqrt{3}-4 \sqrt{5} & \left(18^{3} \times 36\right)
\end{array}
$$

These fitting ranges are determined by $\chi^{2} / d f$ of the fits and the stability of the fitted values of $\alpha$ and $\sigma$. Note that the changes of the fitting ranges are consistent with the change of the scale between $\beta=\beta_{c}\left(N_{t}=4\right)$ and $\beta_{c}\left(N_{t}=6\right)$.

The values for $V(R)$ are plotted in Fig. 2, which are excellently fitted by the rotational invariant fitting curve, Eq.3. The averaged deviation is less than $0.5 \%$ on the both lattices. The results of $V_{0}$, $\alpha, \sigma$ are summarized in Table 2 . 
Table 2

\begin{tabular}{cccc} 
Results of $V_{0}, \alpha, \sigma$ & & \\
\hline$N_{t}$ & $V_{0}$ & $\alpha$ & $\sigma$ \\
4 & $0.630(20)$ & $0.295(14)$ & $0.1493(25)$ \\
6 & $0.627(18)$ & $0.297(19)$ & $0.0655(12)$ \\
\hline
\end{tabular}
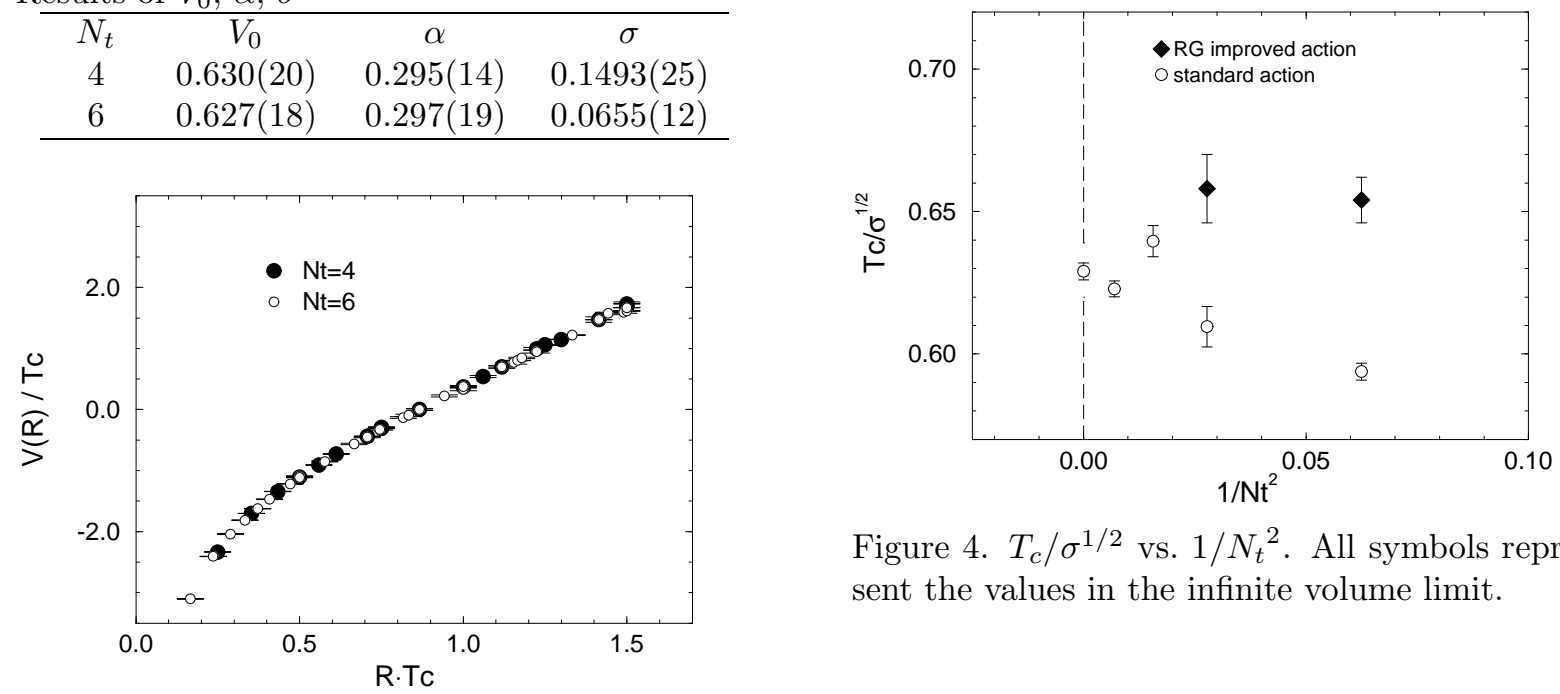

Figure $4 . T_{c} / \sigma^{1 / 2}$ vs. $1 / N_{t}^{2}$. All symbols represent the values in the infinite volume limit.

Figure $3 . \quad V / T_{c}$ vs. $R \cdot T_{c}$. The constant term in the potential is fixed so that both curves have the same value at $R \cdot T_{c}=0.866$.

\section{Scaling properties}

In Fig. 3, the values of $V(R) / T_{c}$ are shown as a function of $R \cdot T_{c}$. We note that the data obtained on the both lattices are in excellent agreement in the whole $R \cdot T_{c}$ region.

We obtain the values of $T_{c} / \sqrt{\sigma}$ in the infinite volume limit:

$T_{c} / \sqrt{\sigma}= \begin{cases}0.647(+7)(5)(3) & \left(N_{t}=4\right) \\ 0.651(+8)(6)(6) & \left(N_{t}=6\right)\end{cases}$

The error in the first bracket is due to the shift of the critical coupling from $\beta_{c}$ at $N_{s} / N_{t}=3$ to the one in the infinite volume limit $\beta_{c}^{\infty}$, the second one is the statistical error and the third one is due to the error in the values of $\beta_{c}^{\infty}$.

The error in the first bracket above has been estimated as follows: We have $\beta_{c}^{\infty}=2.2889(29)$ and $2.5219(52)$ for $N_{t}=4$ and 6 lattices, respectively, assuming both for $N_{t}=4$ and 6 lattices a finite size scaling relation 化 obtained from the data of $\beta_{c}$ on the $12^{3} \times 4$ and $16^{3} \times 4$ lattices: $\beta_{c}\left(N_{t}, \infty\right)=$ $\beta_{c}\left(N_{t}, V\right)+0.168(121) \cdot N_{t}^{3} / V$. Then the values of the string tension at $\beta_{c}^{\infty}$ are estimated assuming an exponential scaling $\sqrt{\sigma}=A \exp (-B \beta)$.

Our results (5) are shown in Fig. 4 together with the results in Ref. [5] using the standard action. Our values are slightly larger than the value extrapolated to the continuum limit with the standard action 0.629(3). Using the average value of (5) and the experimental value $\sigma=$ $(420 \mathrm{MeV})^{2}$, we estimate $T_{c} \sim 276(3)(2) \mathrm{MeV}$. We note that $a^{-1} \approx 1.10 \mathrm{GeV}$ and $1.66 \mathrm{GeV}$, respectively, in the $N_{t}=4$ and 6 cases, when we use the value of $T_{c}$ in $a^{-1}=N_{t} T_{c}$.

Numerical simulations are performed with $\mathrm{Fu}-$ jitsu VPP500/30 and HITAC H6080-FP12 at the University of Tsukuba. This work is in part supported by the Grants-in-Aid of Ministry of Education, Science and Culture (Nos.07NP0401, 07640375 and 07640376).

\section{REFERENCES}

1. T. DeGrand et al., Nucl. Phys. B454 (1995) 615; G.P. Lepage, Nucl. Phys. B (Proc. Suppl.) 47 (1996) 3.

2. Y. Iwasaki, Nucl. Phys. B258 (1985) 141; Univ. of Tsukuba report UTHEP-118 (1983), unpublished.

3. G.S. Bali and K. Schilling, Phys. Rev. D46 (1992) 2636.

4. Y. Iwasaki et al., Phys. Rev. D46 (1992) 4657.

5. G. Boyd et al., Univ. Bielefeld report BI-TP96-04 (hep-lat/9602007). 